\title{
Mechanische Eigenschaften von Human- und Schweineperikard
}

\author{
Naunin, M.; Aliabadi-Zadeh, S.; Barbenel, J.C.; Kraft, M.; Boenick, U. \\ Institut für Mikrotechnik und Medizintechnik, Technische Universität Berlin \\ Bioengineering Unit, University of Strathclyde, Glasgow, UK
}

\section{EINLEITUNG}

In der Vergangenheit hat es umfangreiche Forschungsarbeiten über die mechanischen Eigenschaften von Rinderperikard gegeben, welches als biologisches Material für künstliche Herzklappen eingesetzt wird. Durch das Auftreten der Krankbeit BSE in britischem Vieh wird heute jedoch nach alternativen Materialien gesucht. Humanes autogenes Perikard verspricht, keine Immunreaktionen auszulösen, die auch bei fixierten Fremdgeweben für eine allmähliche Kalzifizierung und damit Versprödung verantwortlich gemacht werden. Schweineperikard ist aufgrund seiner unbegrenzten Verfuigbarkeit interessant.

Eingehende Untersuchungen wurden vor allem mit verschiedenem tierischen Perikard durchgeführt. Die Testmethoden teilen sich in zwei Hauptgruppen mit uniaxialen bzw. biaxialen mechanischen Belastungen. Uniaxiale Zugversuche [1,2] liefern grundlegende einfach reproduzierbare Daten, wohingegen biaxiale Belastungen [3] als physiologischer angesehen werden, jedoch meist eine Reihe weiterer Parameter erfordern und einen komplexen Versuchsaufbau haben.

Als einzige verglichen Zioupos et al. $[4,5,6]$ die Ergebnisse aus uni- und biaxialen Versuchen miteinander. Für das untersuchte Rinderperikard identifizierten sie ein Gebiet uniformer Dicke, das anisotrope mechanische und optische Eigenschaften hatte. Die dabei angewandten Methoden sind auch Grundlage dieser Untersuchung.

Einheitlich wurde das Spannungs- Dehnungsverhalten von Perikard als zeitabhängig und deutlich nicht-linear beschrieben. Über das Vorhandensein richtungsabhängiger Eigenschaften besteht keine Einigkeit.

\section{VERSUCHSAUFBAU UND -DURCHFÜHRUNG}

Je sieben Gewebeproben von humanem (Lebensalter 40-75 Jahre) und tierischem Perikard (Schlachtschweine, 6 Monate alt, $65 \mathrm{~kg}$ ) wurden untersucht. Die anatomische Orientierung war durch Kennzeichnung der Herzachse dargestellt. Einige der Gewebeproben sind vor den Versuchen tiefgekühlt gelagert worden. Bei allen Arbeitsschritten war für eine ausreichende Befeuchtung gesorgt. Um eine Vorkonditionierung für jeweils nachfolgende Tests auszuschließen, wurde das Gewebe in einem Bad mit 50\%iger Ringerlösung relaxiert.

Zunächst mußte das Perikard von anlagerndem Fettgewebe befreit werden. Anschließend war ein Bereich uniformer Dicke zu identifizieren, der für die Verwendung in Herzklappen geeignet wäre.
Um eine Richtungsabhängigkeit der mechanischen Eigenschaften zu ermitteln, wurde das Gewebe in eine Vorrichtung mit kreisförmiger Öffnung gespannt und mit Druckluft beaufschlagt. Die Oberflächenform der entstehenden Blase konnte dann mit der Moiré-Methode beobachtet werden. Hierbei erzeugte die Superposition eines Gitters über der Blase mit ihrem eigenen Schatten auf der Oberfläche des Gewebes Konturlinien. Ovale Konturlinien deuteten auf unterschiedliche Dehnbarkeit in den verschiedenen Richtungen des Gewebes hin, wobei die längere Achse markiert wurde.

Je zwei $2 \mathrm{~mm}$ breite Streifen aus dem zuvor aufgeblähten Bereich wurden in den Orientierungen $0^{\circ}$, $10^{\circ}, 45^{\circ}$ und $90^{\circ}$ in uniaxialen Zugversuchen getestet (längere Achse der Ovale $=90^{\circ}$ ). Die Ausgangslänge der Perikardstreifen betrug $15 \mathrm{~mm}$ und die Streckgeschwindigkeit $10 \mathrm{~mm} / \mathrm{min}$. Die Tests fanden in einem Bad mit 50\%iger Ringerlösung bei Körpertemperatur $\left(37^{\circ} \mathrm{C}\right)$ statt.

Von besonderem Interesse waren die absolute Zugfestigkeit, der terminale Spannungsgradient, welcher der Steigung des Graphen in der linearen Phase entspricht, sowie die Übergangsdehnung, die den Dehnungswert beschreibt, bei dem der nach unten verlängerte lineare Bereich die $\mathrm{x}$-Achse kreuzt.

Gewebe aus den Winkeln zwischen den geschnittenen Streifen wurde mit polarisiertem Licht mikroskopisch untersucht. Diese Technik macht die Orientierung der lastaufnehmenden collagenen Fasern sichtbar.

\section{ERGEBNISSE}

Die Dickemessungen ergaben einen relativ gleichförmigen Bereich auf der Front des Herzens leicht zur anatomisch linken Seite verschoben. Die mittlere Dicke dieser Gebiete lag zwischen 0,26 und 0,44 mm (Mittelwert 0,36 mm mit einer Standardabweichung von $\pm 0,07 \mathrm{~mm}$ ) für menschliches Perikard. Die entsprechenden Werte für das Schweinegewebe lagen bei 0,07 bis $0,18 \mathrm{~mm}(0,12 \pm 0,03 \mathrm{~mm})$.

Das Muster aus der Moire-Methode bei den Inflationstests ergab für die meisten Proben ovale Strukturen und zeigte damit eine Anisotropie an, deren Richtung jedoch sehr stark variierte. Problematisch erwies sich hierbei jedoch der Einfluß durch die notwendige Anpassung des physiologisch gewölbten Perikards an die planare Unterlage.

Das Spannungs-Dehnungsverhalten aus den uniaxialen Zugversuchen war deutlich nicht-linear (Abb. 1). Nach einer sehr nachgiebigen Phase (1), in der kleine Spannungen große Dehnungen hervorriefen, folgte eine 
allmähliche Versteifung (2), die in einen nahezu linearen Bereich (3) überging. An dessen Ende fiel der Gradient (4) und fuhrte zum Versagen. Es wurden verschiedene Formen des Versagens beobachtet. Nur zwei Streifen versagten abrupt. In allen anderen Fällen war ein teilweises Versagen (5) mit anschließendem Fließen (6) bis zum völligen Versagen oder sofort einsetzendes Fließen zu beobachten. Einige Proben zeigten nach partiellem Versagen eine erneute Lastaufnahme, die die erste überstieg. Dies kann durch das Übernehmen anderer lastübertragender Fasern nach dem Versagen der vorigen erklärt werden.

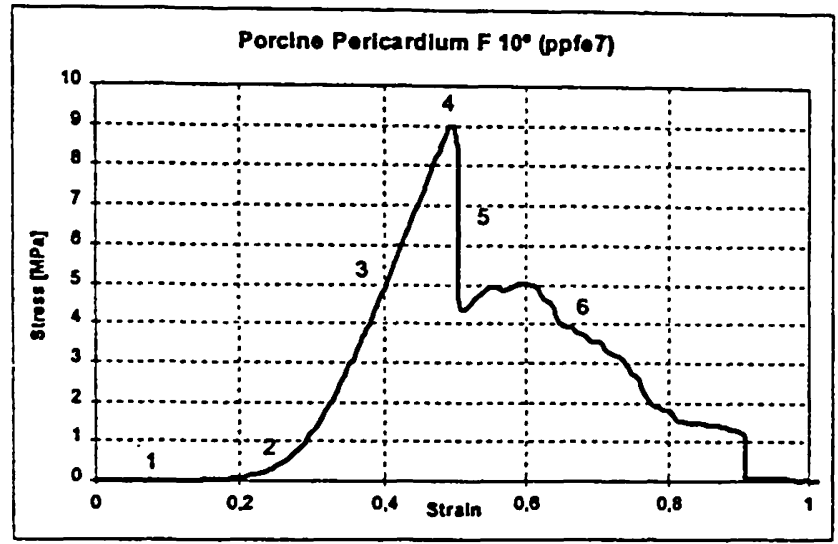

Abb. 1: Spannungs- Dehnungsverhalten von Perikard

Die Versuche ergaben eine weite Streuung der Werte sogar bei direkt nebeneinander liegenden Streifen der selben Richtung. Insbesondere im Hinblick auf die am auffälligsten betroffene Übergangsdehnung könnte dies mit eventueller Faltenbildung oder ungewollter Vordehnung erklärt werden. Bei den Tests mit menschlichem Perikard lag die absolute Zugfestigkeit zwischen 1,0 und 11,4 MPa und der terminale Spannungsgradient (aus Phase 3 des Graphen) von 8,6 bis $84,3 \mathrm{MPa}$. Das Schweineperikard war fester und steifer mit Zugfestigkeiten zwischen 6,6 und 40,1 MPa und Gradienten von 30,2 bis 207,6 MPa. Diese beiden Größen zeigten eine signifikante Korrelation $(p<0,05)$, wobei fuir menschliches Perikard ungefähr absolute Zugfestigkeit $=0,13 *$ terminaler Spannungsgradient galt (Abb. 2). Bei Schweineperikard war der Faktor rund 0,19. Die statistische Analyse ergab keine signifikante Anisotropie für die meisten Gewebeproben. Auch war kein Trend der Werte erkennbar, wenn sie nach der anatomischen Orientierung oder den mikroskopischen Ergebnissen statt nach der Blasenform bei der Aufblähung aufgetragen wurden.

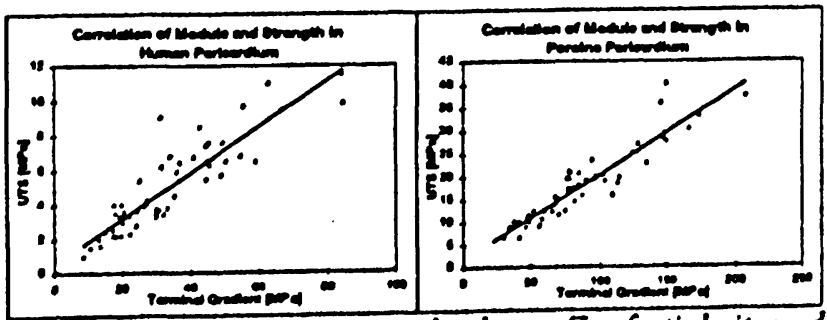

$A b b$. 2: Korrelation von absoluter Zugfestigkelt und terminalem Spannungsgradienten
Die Mikroskopie mit polarisiertem Licht erwies sich als geeignete Methode, die Richtung von kollagenen Fasern zerstörungsfrei zu ermitteln. Es ergaben sich jedoch selbst auf kleinem Raum schon deutliche Richtungsänderungen.

\section{DISKUSSION UND SCHLUBFOLGERUNGEN}

Es konnte gezeigt werden, daß sowohl Human- als auch Schweineperikard einen ausreichend großen Bereich von geeigneter Dicke und Gleichförmigkeit für die Herstellung von künstlichen Herzklappen aufweisen, jedoch die Gleichförmigkeit nicht an die von Rinderperikard $[4,5,6]$ heranreicht.

Eine Anisotropie, wie sie für Rinderperikard gilt, konnte nicht nachgewiesen werden, was auf die erheblich kleineren Abmessungen der Herzen von Mensch und Schwein rückführbar ist. Die im kleineren Maßstab möglicherweise anisotropen Eigenschaften waren bei der gewählten Probengröße nicht mehr nachweisbar. Diese Annahme konnte mikroskopisch bestätigt werden. Dies erklärt auch die starke Streuung der Werte.

Der Zusammenhang zwischen dem terminalen Spannungsgradienten und der absoluten Zugfestigkeit ist mit der Anzahl kraftübertragender Fasern in Streifenrichtung pro Volumeneinheit erklärbar.

Die Untersuchung machte auch deutlich, daß eine Extrapolation von Fakten einer Spezies auf eine andere nur mit Vorsicht gemacht werden sollte, da teilweise deutliche Variationen bestehen.

Aus Sicht der mechanischen Eigenschaften erweist sich Schweineperikard im Vergleich zu menschlichem Gewebe als günstigeres Ersatzmaterial für Rinderperikard bei der Konstruktion von künstlichen Herzklappen. Die Abwesenheit von Anisotropie kann auch als Vorteil gesehen werden, da heutige Herzklappenkonstruktionen von einem isotropen Gewebe ausgehen.

\section{LITERATUR}

[1] Lee, J.M., Boughner, D.R., (1981) "Tissue Mechanics of Canine Pericardium in Different Test Environments", Circ. Res., 49, pp. 533-544

[2] Lee, J.M., Boughner, D.R., (1985) "Mechanical Properties of Human Pericardium", Circ. Res., 57, pp. 475-481

[3] Lee, M.-C., Le Winter, M.M., Freeman, G., Shabetal, R., Fung, Y.C., (1985) "Biaxial mechanical properties of the pericardium in normal and volume overload dogs", Am. J. Physiol., 249, (Heart Circ. Physiol., 18) pp. H222-H230

[4] Zioupos, P.N., (1989) "Mechanical properties and structure of bovine pericardium", Ph.D. Thesis, University of Strathclyde, Glasgow, UK

[5] Zioupos, P.N., Barbenel, J.C., Fisher, J., (1992) "Mechanical and optical anisotropy of bovine pericardium", Med.\&Biol.Eng.\&Comput., 30, pp.76-\$2 [6] Zioupos, P.N., Barbenel, J.C., (1994), "Mechanics of native bovine pericardium (I and II)", Biomaterials, Vol. 15 No. 5, pp. 366-382 